\title{
ON THE SURJECTIVITY OF LINEAR MAPS ON LOCALLY CONVEX SPACES
}

\author{
SADAYUKI YAMAMURO \\ (Received 23 September 1980) \\ Communicated by R. Vyborny
}

\begin{abstract}
The aim of this note is to investigate the structure of general surjectivity problem for a continuous linear map between locally convex spaces. We shall do so by using the method introduced in Yamamuro (1980). Its basic notion is that of calibrations which has been introduced in Yamamuro (1975), studied in detail in Yamamuro (1979) and appliced to several problems in Yamamuro (1978) and Yamamuro (1979a).
\end{abstract}

1980 Mathematics subject classification (Amer. Math. Soc.): 46 A 05.

We shall start this note with a few words about the calibrations.

Let $E$ and $F$ be Hausdorff locally convex linear spaces. A semi-norm map on $(E, F)$ is a map $p$ whose values $p_{E}$ and $p_{F}$ are continuous semi-norms on $E$ and $E$ respectively. Let $\Gamma$ be a set of semi-norm maps on $(E, F)$ and set

$$
\Gamma_{E}=\left\{p_{E}: p \in \Gamma\right\} \text { and } \Gamma_{F}=\left\{p_{F}: p \in \Gamma\right\} \text {. }
$$

A calibration for $(E, F)$ is a set $\Gamma$ of semi-norm maps on $(E, F)$ such that $\Gamma_{E}$ and $\Gamma_{F}$ induce the topologies of $E$ and $F$ respectively.

When $\Gamma$ is a calibration for $(E, F)$, a linear map $u: E \rightarrow F$ is said to be $\Gamma$-continuous if, for each $p \in \Gamma$,

$$
p(u)=p_{(E, F)}(u)=\sup \left\{p_{F}[u(x)]: p_{E}(x)<1\right\}<+\infty .
$$

The set of all $\Gamma$-continuous linear maps of $E$ into $F$ is denoted by $L_{\mathrm{T}}(E, F)$.

It was shown in Yamamuro (1979), page 11, that, for any continuous linear map $u: E \rightarrow F$, there exists a calibration $\Gamma$ for $(E, F)$ such that $u \in L_{\mathrm{T}}(E, F)$. Naturally, it is desirable to choose such a calibration which reflects the full

Copyright Australian Mathematical Society 1982 
nature of the given map. It will then amount to characterizing the map in terms of calibrations. We find various results of such nature in Treves (1967), where Treves successfully generalized some theorems in the theory of partial differential equations to those for linear maps on locally convex spaces.

In a series of papers, R. Mennicken and B. Sagraloff have generalized some results of Treves (see, for example, Mennicken and Sagraloff (1980)).

We shall start with very basic facts about the $\Gamma$-completions of a locally convex space with respect to a calibration $\Gamma$. When $\Gamma$ is a calibration for $(E, F)$, we shall have two families $\{E[p]: p \in \Gamma\}$ and $\{F[p]: p \in \Gamma\}$ of Banach spaces, and a continuous linear map $u: E \rightarrow F$ will be extended to a continuous linear map $u_{p}: E[p] \rightarrow F[p]$ for every $p \in \Gamma$. The first step of our study is to characterize the surjectivity of $u$ by the properties of these maps between Banach spaces. We shall then reduce this general criterion to more convenient forms and conclude this note with a generalization of a theorem of M. Eidelheit, which will then lead us to the Colombeau's version of a theorem of E. Borel.

\section{1. r-completions of locally convex spaces}

Let $E$ be a vector space and $p$ be a semi-norm on $E$. A sequence $\left(x_{i}\right)$ in $E$ is said to be $p$-Cauchy if $p\left(x_{i}-x_{j}\right) \rightarrow 0$ as $i, j \rightarrow \infty$. Two $p$-Cauchy sequences $\left(x_{i}\right)$ and $\left(y_{i}\right)$ are said to be equivalent if $p\left(x_{i}-y_{i}\right) \rightarrow 0$ as $i \rightarrow \infty$.

Let $\left(x_{i}\right)$ be a $p$-Cauchy sequence and $\mathbf{a}$ be the set of all $p$-Cauchy sequences in $E$ which are equivalent to $\left(x_{i}\right)$. Such a set a is called a $p$-class on $E$. Obviously, if two $p$-classes have a common element, they must be identical.

The set of all $p$-classes on $E$ will be denoted by $E[p]$ and it will be called the $p$-completion of $E$. It is a vector space when $\alpha \mathbf{a}+\beta \mathbf{b}$ is defined to be the $p$-class which contains the sequence $\left(\alpha x_{i}+\beta y_{i}\right)$ for some $\left(x_{i}\right) \in \mathbf{a}$ and $\left(y_{i}\right) \in \mathbf{b}$. The zero element of $E[p]$ is, therefore, the $p$-class which contains a $p$-null sequence.

For $\mathbf{a} \in E[p]$, we define

$$
p(\mathbf{a})=\lim _{i \rightarrow \infty} p\left(x_{i}\right) \quad \text { for }\left(x_{i}\right) \in \mathbf{a} .
$$

Then, the value $p(a)$ does not depend on the choice of $\left(x_{i}\right)$ from a. It is obvious that $p$ is a norm on $E[p]$ and, with this norm, $E[p]$ is a Banach space.

For each $x \in F$, let $S_{p}(x)$ be the element of $E[p]$ which contains the $p$-Cauchy sequence all of whose terms are identical to $x$. Then, we have

$$
p\left[S_{p}(x)\right]=p(x) \text { for every } x \in E .
$$


For $\mathbf{a} \in E[p]$ and $\left(x_{i}\right) \in \mathbf{a}$, we have

$$
\lim _{i \rightarrow \infty} p\left[S_{p}\left(x_{i}\right)-\mathbf{a}\right]=0,
$$

which shows that $S_{p}(E)$ is dense in $E[p]$.

We shall use the following notations:

$$
\begin{aligned}
U_{E}(p, \alpha) & =\{x \in E: p(x)<\alpha\}, & & B_{E}(p, \alpha)=\{x \in E: p(x)<\alpha\}, \\
U_{E}[p, \alpha] & =\{\mathbf{a} \in E[p]: p(\mathbf{a})<\alpha\}, & B_{E}[p, \alpha] & =\{\mathbf{a} \in E[p]: p(\mathbf{a})<\alpha\} .
\end{aligned}
$$

We also denote the corresponding unit spheres by $U_{E}(p), B_{E}(p), U_{E}[p]$ and $B_{E}[p]$ respectively.

Let $E$ be a locally convex space and $\Gamma$ be a calibration for $E$. Then, for each $p \in \Gamma$, we have the $p$-completion $E[p]$ of $E$. The family $\{E[p]: p \in \Gamma\}$ of Banach spaces will be called the $\Gamma$-completion of $E$.

Thus, we have a projective system:

$$
S_{p}: E \rightarrow E[p] \text { for all } p \in \Gamma \text {. }
$$

It is easy to see that the projective topology on $E$ defined by this system coincides with the topology of $E$.

When $p>q$, that is, $p(x)>q(x)$ for all $x \in E$, we have a natural embedding:

$$
T_{p, q}: E[p] \rightarrow E[q]
$$

which maps every $\mathbf{a} \in E[p]$ to the $q$-class which contains elements of a. Obviously, this map is linear,

$$
q\left[T_{p, q}(\mathbf{a})\right] \leqslant p(\mathbf{a}) \quad \text { for every } \mathbf{a} \in E[p]
$$

and

$$
T_{p, q} \circ S_{p}=S_{q}
$$

Furthermore, it is evident that $T_{p, q}(E[p])$ is dense in $E[q]$.

(1.1). E is complete if and only if the following condition is satisfied: if $\mathbf{a}_{p} \in E[p]$ for all $p \in \Gamma$ and

$$
T_{p, q}\left(\mathrm{a}_{p}\right)=\mathrm{a}_{q} \text { whenever } p \geqslant q,
$$

then there exists $x \in E$ such that $S_{p}(x)=\mathrm{a}_{p}$ for all $p \in \Gamma$.

For the proof, see Köthe (1967), page 231 and page 232.

We shall have occasion to use the notion of gauged calibrations introduced in Yamamuro (1980), page 434. A calibration $\Gamma$ for a locally convex space $E$ is said to be gauged if $\Gamma$ is directed and there exists a net $\gamma: \Gamma \rightarrow R$ (the reals) such that $\gamma(p) \rightarrow 0$. This function $\gamma$ is called a gauge of $\Gamma$. 


\section{A general surjection theorem}

Let $\Gamma$ be a calibration for $(E, F)$. For each $u \in L_{\Gamma}(E, F)$, we have a continuous extension

$$
u_{p}: E[p] \rightarrow F[p],
$$

where $E[p]=E\left[p_{E}\right]$ and $F[p]=F\left[p_{F}\right]$, and the following diagram commutes:

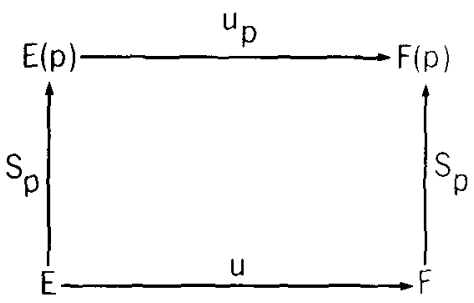

A linear map $u \in L_{\mathrm{T}}(E, F)$ is said to be $S$-resonant (with respect to $\Gamma$ ) if the following condition is satisfied: if $y \in F$ and there exist $a_{p} \in E[p]$ such that $u_{p}\left(\mathrm{a}_{p}\right)=S_{p}(y)$ for all $p \in \Gamma$, then there exists $x \in E$ such that $y=u(x)$.

A linear map $u \in L_{\mathrm{T}}(E, F)$ is said to be $p$-resonant for some $p \in \Gamma$ if the following condition is satisfied: if $\left(u\left(x_{i}\right)\right)$ is a $p_{F}$-Cauchy sequence, there exists a $p_{E}$-Cauchy sequence $\left(z_{i}\right)$ such that $p_{F}\left[u\left(x_{i}\right)-u\left(z_{i}\right)\right] \rightarrow 0$.

If $u$ is $p$-resonant for every $p \in \Gamma$, it is said to be $\Gamma$-resonant.

All these definitions have been given in Yamamuro (1980).

(2.1). For $u \in L_{\Gamma}(E, F)$, the following two conditions are equivalent.

(1) $u$ is surjective and $\Gamma$-resonant;

(2) every $u_{p}$ is surjective and $u$ is $S$-resonant.

This is implied by the following four statements.

(2.2). If $u_{p}$ is surjective, then $u$ is p-resonant.

Proof. Assume that $\left(u\left(x_{i}\right)\right)$ is $p_{F}$-Cauchy and let $\mathbf{b} \in F[p]$ be the $p_{F}$-class which contains it. Then, $u_{p}(\mathbf{a})=\mathbf{b}$ for some $\mathbf{a} \in E[p]$ and, for any $\left(z_{i}\right) \in \mathbf{a}$, we have $p_{F}\left[u\left(x_{i}\right)-u\left(z_{i}\right)\right] \rightarrow 0$ as $i \rightarrow \infty$.

(2.3). If every $u_{p}$ is surjective and $u$ is $S$-resonant, $u$ is surjective.

Proof. For any $y \in F$, since $S_{p}(y) \in F[p]$ and $u_{p}$ is surjective, there exists $\mathbf{a}_{p} \in E[p]$ for every $p \in \Gamma$ such that $u_{p}\left(a_{p}\right)=S_{p}(y)$. Hence, by the $S$-resonance, $y=u(x)$ for some $x \in E$. 
(2.4). If $u$ is surjective, $u$ is $S$-resonant.

The proof is trivial.

(2.5). If $u(E)$ is dense in $F$ and $u$ is p-resonant, $u_{p}$ is surjective.

Proof. The $p$-resonance is equivalent to

$$
u_{p}(E[p]) \supset \overline{S_{p}(u(E))} \text {. }
$$

Hence,

$$
F[p]=\overline{S_{p}(F)}=\overline{S_{p}(\overline{u(E)})}=\overline{S_{p}(u(E))} \subset u_{p}(E[p]) .
$$

EXAMPLE 1. Let $E$ be a locally convex space equipped with a calibration $\Gamma_{E}$ and let $M$ be a closed linear subspace of $E$. We consider the quotient

$$
\phi: E \rightarrow E / M \text {. }
$$

For each $p_{E} \in \Gamma_{E}$, we set

$$
p_{E / M}(\phi(x))=\inf \left\{p_{E}(z): \phi(x)=\phi(z)\right\} .
$$

Then, $\left\{p_{E / M}: p_{E} \in \Gamma_{E}\right\}$ defines the quotient topology on $E / M$. The calibration

$$
\Gamma=\left\{p=\left(p_{E}, p_{E / M}\right): p_{E} \in \Gamma_{E}\right\}
$$

is called the quotient calibration for $(E, E / M)$ defined from $\Gamma_{E}$. Then, $\phi \in$ $L_{\mathrm{T}}(E, E / M), \phi$ is obviously surjective and it is easy to show that $\phi$ is $\Gamma$ resonant.

EXAMPLe 2. Let $E$ be a locally convex space equipped with a calibration $\Gamma_{E}$ and let $\tilde{E}$ be the completion of $E$. Hence, there exists an embedding

$$
\psi: E \rightarrow \tilde{E}
$$

such that $\psi(E)$ is dense in $\tilde{E}$. For each $p_{E} \in \Gamma_{E}$, its extension $\tilde{p}_{E}$ over $\tilde{E}$ is defined and $\left\{\tilde{p}_{E}: p_{E} \in \Gamma_{E}\right\}$ defines the locally convex topology on $\tilde{E}$. The calibration

$$
\Gamma=\left\{p=\left(p_{E}, \tilde{p}_{E}\right): p_{E} \in \Gamma_{E}\right\}
$$

is called the completion calibration for $(E, \tilde{E})$. Since

$$
\tilde{p}_{E}(\psi(x))=p_{E}(x) \text { for every } x \in E \text { and } p \in \Gamma,
$$

$\psi$ is $\Gamma$-resonant. Hence, every $\psi_{p}$ is surjective.

EXAMPLE 3. Let $E$ be a complete locally convex space equipped with a calibration $\Gamma_{E}$ and assume that there exists a closed linear subspace $M$ of $E$ such that $E / M$ is not complete. Let $F$ be the completion of $E / M$. For each $p_{E} \in \Gamma_{E}$, 
we form the quotient semi-norm $p_{E / M}$ as in Example 1 and, then, extend it as in Example 2 to a semi-norm $p_{F}$ on $F$. Then, we have a calibration

$$
\Gamma=\left\{p=\left(p_{E}, p_{F}\right): p_{E} \in \Gamma_{E}\right\}
$$

for $(E, F)$. Then, the map

$$
\psi \circ \phi: E \rightarrow F
$$

between complete spaces belongs to $L_{\mathrm{T}}(E, F)$. Every $(\psi \circ \phi)_{p}$ is surjective, but $\psi \circ \phi$ is not surjective.

When a pair $(E, F)$ is equipped with a gauged calibration, we have the converse to (2.5) as follows.

(2.6). Assume that $\Gamma$ is a calibration for $(E, F)$ and $u \in L_{\Gamma}(E, F)$. If $\Gamma_{F}$ is gauged, the following two conditions are equivalent:

(1) every $u_{p}$ is surjective;

(2) $u(E)$ is dense in $F$ and $u$ is $\Gamma$-resonant.

Proof. In view of (2.2) and (2.5), we only need to show that (1) implies $\overline{u(E)}=F$. Assume that every $u_{p}$ is surjective and $y \in F$. Then, for each $p \in \Gamma$, there exists a $p_{E}$-Cauchy sequence $\left(x_{p, i}\right)$ such that

$$
p_{F}\left(y-u\left(x_{p, i}\right)\right) \rightarrow 0 \text { as } i \rightarrow \infty .
$$

Hence, there exists $\left(i_{p}\right)$ such that

$$
p_{F}\left(y-u\left(x_{p, i_{p}}\right)\right)<\gamma_{F}(p),
$$

where $\gamma_{F}$ is a gauge of $\Gamma_{F}$. Then, we have $u\left(x_{p, i_{p}}\right) \rightarrow y$.

EXAMPLE 4. To show that (2.6) does not necessarily hold when $\Gamma_{F}$ is not gauged, we consider the case when $E$ is a Banach space equipped with the weak topology. Let $E^{\prime}$ be its dual and, for a non-zero element $a^{\prime} \in E^{\prime}$, we set

$$
\Gamma_{w}=\left\{\left|x^{\prime}\right|: x^{\prime} \in E^{\prime},\left|x^{\prime}\right| \geqslant\left|a^{\prime}\right|\right\},
$$

where $\left|x^{\prime}\right|$ is a semi-norm defined by

$$
\left|x^{\prime}\right|(x)=\left|x^{\prime}(x)\right| \text { for every } x \in E .
$$

Let $F$ be the space $E$ equipped with the calibration $\Gamma_{w}$ and let $\Gamma$ be a calibration for $(E, F)$ defined by

$$
\Gamma=\left\{\left(\|\cdot\|,\left|x^{\prime}\right|\right):\left|x^{\prime}\right| \in \Gamma_{w}\right\} .
$$

Let $a \in E$ be such that $a^{\prime}(a) \neq 0$ and we define $u: E \rightarrow F$ by

$$
u(x)=a^{\prime}(x) a \text { for every } x \in F \text {. }
$$


Then, for $p=\left(\|\cdot\|,\left|x^{\prime}\right|\right)$,

$$
\begin{aligned}
p_{F}(u(x)) & =\left|x^{\prime}\right|\left(a^{\prime}(x) a\right)=\left|a^{\prime}(x) x^{\prime}(a)\right| \\
& \leqslant\left|x^{\prime}(a)\right| \cdot\left\|a^{\prime}\right\| \cdot p_{E}(x),
\end{aligned}
$$

which shows that $u \in L_{\Gamma}(E, F)$. Now, for $p=\left(\|\cdot\|,\left|x^{\prime}\right|\right) \in \Gamma$, assume that $\mathbf{b} \in F[p]$ and $\left(y_{i}\right) \in \mathbf{b}$; then $\left(y_{i}\right)$ is $\left|x^{\prime}\right|$-Cauchy and, hence, $\left(x^{\prime}\left(y_{i}\right)\right)$ converges to a number $\eta$. By the definition of $\Gamma_{w}$, we have $x^{\prime}(a) \neq 0$. Hence, we can choose $x \in E$ such that

$$
a^{\prime}(x) x^{\prime}(a)=\eta
$$

which shows

$$
\left|x^{\prime}\right|\left(y_{i}-u(x)\right)=\left|x^{\prime}\left(y_{i}\right)-\eta\right| \rightarrow 0 \text { as } i \rightarrow \infty .
$$

Thus, we have $u_{p}\left(S_{p}(x)\right)=\mathbf{b}$, or, every $u_{p}$ is surjective. However, $u(E)$ is a one-dimensional subspace of $E$.

A similar proof as that of (2.6) gives the following fact.

(2.7). Assume that $\Gamma$ is a calibration for $(E, F)$ and $u \in L_{T}(E, F)$. If $\Gamma_{F}$ is gauged and $u(E)$ is closed, then $u$ is $S$-resonant.

When $E$ and $F$ are Banach spaces and $\Gamma$ consists of a single norm map whose values are norms of these spaces, any continuous linear map of $E$ into $F$ is $S$-resonant. Therefore, the $S$-resonance does not always imply that $u(E)$ is closed even if $\Gamma_{F}$ is gauged. Furthermore, for the map $u$ in Example 4, we see that $u(E)$ is closed and $u$ is not $S$-resonant. Therefore, (2.7) is not always true if $\Gamma_{F}$ is not gauged.

\section{Compatibility with completions}

Let $\Gamma$ be a calibration for $(E, F)$ and $u \in L_{\Gamma}(E, F)$. We start with another criterion for the $S$-resonance.

(3.1). Assume that $E$ is complete. If every $u_{p}$ is injective, then $u$ is $S$-resonant.

Proof. Let $\mathrm{a}_{p} \in E[p]$ for all $p \in \Gamma$ and $y \in F$ such that

$$
u_{p}\left(a_{p}\right)=S_{p}(y) \text { for all } p \in \Gamma \text {. }
$$

Then, if $p>q$,

$$
u_{q}\left(\mathbf{a}_{q}\right)=S_{q}(y)=T_{p, q} \circ S_{p}(y)=T_{p, q} \circ u_{p}\left(\mathrm{a}_{p}\right)=u_{q} \circ T_{p, q}\left(\mathrm{a}_{p}\right) .
$$


Since $u_{q}$ is injective, we have

$$
\mathbf{a}_{q}=T_{p, q}\left(\mathbf{a}_{p}\right) \quad \text { whenever } p>q,
$$

and, by (1.1), there exists $x \in E$ such that $S_{p}(x)=\mathrm{a}_{p}$ for every $p \in \Gamma$, which implies $y=u(x)$.

In this section, we shall be concerned with the relation between the injectivity of $u$ and that of $u_{p}$. It is evident that, if every $u_{p}$ is injective, $u$ is injective, but the converse is not true.

As in Example 1, we shall consider the quotient

$$
\phi: E \rightarrow \hat{E}=E / M,
$$

where $M=\operatorname{ker}(u)$. Each $p \in \Gamma$ defines

$$
\hat{p}_{E}(\phi(x))=\inf \left\{p_{E}(z): u(x)=u(z)\right\},
$$

and $\Gamma$ can be regarded as a calibration for the family $\{E, \hat{E}, F\}$, where $p_{\hat{E}}=\hat{p}_{E}$ for every $p \in \Gamma$. The linear map $u$ defines an injective linear map $\hat{u}: \hat{E} \rightarrow F$ defined by $u=\hat{u} \circ \phi$ and it is obvious that $\hat{u} \in L_{\mathrm{T}}(\hat{E}, F)$. Furthermore, for the spaces $E, \hat{E}$ and $F$, there are $\Gamma$-completions $\{E[p]\},\{\hat{E}[p]\}$ and $\{F[p]\}$. Then, the following diagram commutes:

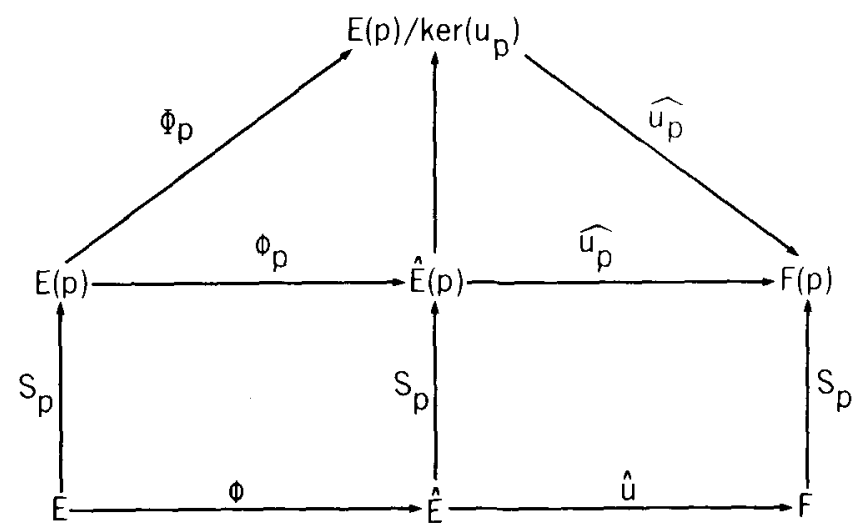

We shall say that $u$ is $p$-compatible if the following condition is satisfied: if $p_{F}\left(u\left(x_{i}\right)\right) \rightarrow 0$ and $\left(x_{i}\right)$ is a $p_{E}$-Cauchy sequence, then there exists $\left(z_{i}\right)$ such that $u\left(x_{i}\right)=u\left(z_{i}\right)$ and $p_{E}\left(z_{i}\right) \rightarrow 0$.

(3.2). For $u \in L_{\mathrm{T}}(E, F)$ and $p \in \Gamma$, the following conditions are equivalent:

(1) $u$ is p-compatible;

(2) $\operatorname{ker}\left(u_{p}\right)=\overline{S_{p}(\operatorname{ker}(u))}$;

(3) $\operatorname{ker}\left(u_{p}\right)=\operatorname{ker}\left(\phi_{p}\right)$; 
(4) $\hat{u}_{p}: \hat{E}[p] \rightarrow F[p]$ is injective;

(5) $p_{\hat{E}}(\phi(x))=p_{\Phi}\left(\phi_{p}\left(S_{p}(x)\right)\right)$ for every $x \in E$, where $p_{\Phi}$ is the quotient norm defined by $p$ on $E[p] / \operatorname{ker}\left(u_{p}\right)$;

(6) the map

$$
K_{p}: \hat{E}[p] \rightarrow E[p] / \operatorname{ker}\left(u_{p}\right):\left(\phi\left(x_{i}\right)\right) \rightarrow\left(\phi_{p} \circ S_{p}\left(x_{i}\right)\right)
$$

is an isomorphic surjection.

Proof. To prove that (1) implies (2), assume that $\left(x_{i}\right) \in \mathbf{a} \in \operatorname{ker}\left(u_{p}\right)$. Then, $p_{F}\left(u\left(x_{i}\right)\right) \rightarrow 0$ and $\left(x_{i}\right)$ is a $p_{E}$-Cauchy sequence. Hence, there exists $\left(z_{i}\right)$ such that $u\left(x_{i}\right)=u\left(z_{i}\right)$ and $p_{E}\left(z_{i}\right) \rightarrow 0$. Then, $x_{i}-z_{i} \in \operatorname{ker}(u)$ and $p_{E}\left(S_{p}\left(x_{i}-z_{i}\right)-\mathbf{a}\right) \rightarrow 0$.

To prove that (2) implies (3), assume that $a \in \operatorname{ker}\left(u_{p}\right)$. Then, there exist $x_{i} \in \operatorname{ker}(u)$ such that $S_{p}\left(x_{i}\right) \rightarrow \mathbf{a}$ in the space $E[p]$. Hence, in the space $\hat{E}[p]$,

$$
\phi_{p}(\mathbf{a})=\lim _{i \rightarrow \infty} \phi_{p}\left(S_{p}\left(x_{i}\right)\right)=\lim _{i \rightarrow \infty} S_{p}\left(\phi\left(x_{i}\right)\right)=0 .
$$

To prove that (3) implies (4), assume that $\hat{u}_{p}(\hat{\mathbf{a}})=0$ for some $\hat{\mathbf{a}} \in \hat{E}[p]$. As was stated in Example 1, $\phi_{p}$ is surjective. Hence, $\hat{\mathbf{a}}=\phi_{p}(\mathbf{a})$ for some $\mathbf{a} \in E[p]$ and $u_{p}(\mathbf{a})=\hat{u}_{p}(\hat{\mathbf{a}})=0$, which implies $\phi_{p}(\mathbf{a})=0$.

To prove that (4) implies (5), assume that $\alpha>p_{\phi}\left(\phi_{p} \circ S_{p}(x)\right)$. Then, by the definition of the quotient norm, there exists $\mathbf{a} \in E[p]$ such that $p_{E}(\mathbf{a})<\alpha$ and $u_{p}\left(\mathbf{a}-S_{p}(x)\right)=0$. Since $u_{p}=\hat{u}_{p} \circ \phi_{p}$ and $\hat{u}_{p}$ is injective, we have $\phi_{p}(\mathbf{a})=$ $\phi_{p}\left(S_{p}(x)\right)$. Therefore,

$$
p_{\hat{E}}(\phi(x))=p_{\hat{E}}\left(S_{p}(\phi(x))\right)=p_{\hat{E}}\left(\phi_{p}\left(S_{p}(x)\right)\right)=p_{\hat{E}}\left(\phi_{p}(\mathbf{a})\right) \leqslant p_{E}(\mathbf{a})<\alpha .
$$

To prove that (5) implies (6), we first note that $K_{p}$ is surjective because $\phi_{p}=K_{p} \circ \phi_{p}$. Furthermore, (5) implies $p_{E}(\mathbf{a})=p_{\phi}\left(K_{p}(\mathbf{a})\right)$ for every $\mathbf{a} \in E[p]$.

To prove that (6) implies (1), assume that $p_{F}\left(u\left(x_{i}\right)\right) \rightarrow 0$ and $\left(x_{i}\right) \in \mathbf{a} \in E[p]$, which means that $u_{p}(\mathrm{a})=0$. Then, since $u_{p}=\hat{u}_{p} \circ K_{p} \circ \phi_{p}$ and $\hat{u}_{p}$ is injective, we have $K_{p} \circ \phi_{p}(a)=0$ and, by $(6), \phi_{p}(a)=0$, which is equivalent to $p_{\hat{E}}\left(\phi\left(x_{i}\right)\right) \rightarrow 0$.

Thus, the $\Gamma$-compatibility ensures that the injective map $\hat{u}: \hat{E} \rightarrow F$ has injective extensions $\hat{u}_{p}: \hat{E}[p] \rightarrow F[p]$ for all $p \in \Gamma$.

(3.3). Assume that $\hat{E}$ is complete. Then, if $u$ is $\Gamma$-compatible, $u$ is $S$-resonant.

Proof. Since all $\hat{u}_{p}$ are injective, (3.1) implies that $\hat{u}$ is $S$-resonant, which is equivalent to that $u$ is $S$-resonant.

When $\Gamma$ is a Fréchet calibration for $(E, F), \hat{E}$ is complete and $\Gamma_{E}$ is obviously gauged. Hence, in this case, the $\Gamma$-compatibility implies the $S$-resonance. It is an 
open question whether every quotient space of a complete locally convex space with a gauged calibration by a closed linear subspace is again complete.

The maps considered in Examples 1, 2, 3 and 4 are all $\Gamma$-compatible and those in Examples 2, 3 and 4 are not $S$-resonant. The $S$-resonance does not imply the $\Gamma$-compatibility in general. If $u: E \rightarrow F$ is surjective, then it is $S$-resonant with respect to any calibration $\Gamma$ such that $u \in L_{\mathrm{T}}(E, F)$, whereas the $\Gamma$-compatibility depends on a particular choice of calibration.

REMARK. The treatment of the Whitney's extension theorem by Malgrange (1966) illustrates a meaning of the above statement (3.3). Let $\Omega$ be an open subset of $R^{m}$ and $E=C^{\infty}(\Omega)$ be the set of all real-valued $C^{\infty}$-functions on $\Omega$. For a sequence $\left(K_{n}\right)$ of compact subsets such that $K_{n} \subset K_{n+1}^{\circ}$ (the interior of $\left.K_{n+1}\right)$ and $\Omega=\cup K_{n}$, we define semi-norms:

$$
q_{k, n}(x)=\sup \left\{\left|x^{(i)}(t)\right|: 0<i<k, t \in K_{n+1}^{\circ}\right\} .
$$

Then, $E$ is a Fréchet space with the calibration $\left\{q_{k, n}: k, n \geqslant 0\right\}$, and it is easy to see that $q_{k, n}$-completion $E\left[q_{k, n}\right]$ of $E$ is the space $C^{k}\left(K_{n+1}^{\circ}\right)$ of all $C^{k}$-functions on $K_{n+1}^{\circ}$. Let $F$ be the space of all Whitney functions $\left\{x^{i}: i>0\right\}$ of class $C^{\infty}$ defined on a fixed compact subset $K$ of $\Omega$. For the definition of these functions, see Malgrange (1966), page 3. This is also a Fréchet space with a calibration consisting of, using his notation at page 4 ,

$$
r_{k}(x)=\|x\|_{k}^{K},
$$

and the $r_{k}$-completion $F\left[r_{k}\right]$ of $F$ is the space of all Whitney functions of class $C^{k}$ defined on $K$. Now, let $u: E \rightarrow F$ be a linear map defined by

$$
u(x)^{i}(t)=x^{(i)}(t) \text { for } i=0,1,2, \ldots \text { and } t \in K .
$$

Then, for the calibration

$$
\Gamma=\left\{p_{k, n}=\left(q_{k, n}, r_{k}\right): k, n \geqslant 0\right\}
$$

and $u_{k, n}=u_{p_{k, n}}$, the fact that all extensions

$$
u_{k, n}: E\left[p_{k, n}\right] \rightarrow F\left[p_{k, n}\right]
$$

are surjective is exactly the Whitney's extension theorem. Lemma 4.3 of Malgrange (1966), page 11, states that

$$
\operatorname{ker}\left(u_{k, n}\right) \subset \overline{S_{k, n}(\operatorname{ker}(u))}
$$

for the imbedding $S_{k, n}=S_{p_{k, n}}$, which means that $u$ is $\Gamma$-compatible. Hence, by (3.3), every Whitney function of class $C^{\infty}$ on $K$ can be extended to a $C^{\infty}$-function on $\Omega$.

A well-known corollary of this theorem, a theorem of E. Borel, will be proved in a different manner in Section 6. 


\section{4. $\Gamma$-open maps}

Let $\Gamma$ be a calibration for $(E, F)$ and $p \in \Gamma$. A continuous linear map $u$ : $E \rightarrow F$ is said to be $p$-open if the following condition is satisfied: if $p_{F}\left(u\left(x_{i}\right)\right) \rightarrow 0$, then there exist $z_{i} \in E$ such that $u\left(x_{i}\right)=u\left(z_{i}\right)$ and $p_{E}\left(z_{i}\right) \rightarrow 0$. If $u$ is $p$-open for every $p \in \Gamma, u$ is said to be $\Gamma$-open.

The above definition is equivalent to that, if $p_{F}\left(u\left(x_{i}\right)\right) \rightarrow 0$, then $p_{\hat{E}}\left(\phi\left(x_{i}\right)\right) \rightarrow 0$, where $\phi: E \rightarrow \hat{E}=E / \operatorname{ker}(u)$ is the canonical map. Hence, $u$ is $p$-open if and only if $\hat{u}: \hat{E} \rightarrow F$ is $p$-open.

We recall that a subset $X$ of $E$ is said to be $p$-open for $p \in \Gamma$ if it is an open subset of the semi-normed space $\left(E, p_{E}\right)$. See Yamamuro (1979), page 18, for this definition and the related topics.

(4.1). Let $\Gamma$ be a calibration for $(E, F)$ and $p \in \Gamma$. For a continuous linear map $u: E \rightarrow F$, the following conditions are equivalent:

(1) $u$ is p-open;

(2) there exists a constant $\alpha_{p}>0$ such that

$$
p_{E}(\phi(x)) \leqslant \alpha_{p} p_{F}(u(x)) \text { for every } x \in E ;
$$

(3) there exists a constant $\alpha_{p}>0$ such that

$$
u(E) \cap U_{F}(p) \subset u\left(U_{E}\left(p, \alpha_{p}\right)\right)
$$

(4) for every p-open subset $X$ of $E, u(X)$ is a p-open subset of $u(E)$.

Proof. To prove that (1) implies (2), assume that $u$ is $p$-open and

$$
\inf \left\{p_{F}(u(x)) / p_{\hat{E}}(\phi(x)): p_{\hat{E}}(\phi(x)) \neq 0\right\}=0 \text {. }
$$

Then, there exist $x_{n} \in E$ such that

$$
p_{F}\left(u\left(x_{n}\right)\right)<n^{-1} p_{\hat{E}}\left(\phi\left(x_{n}\right)\right),
$$

and, for $\alpha_{n}=p_{\hat{E}}\left(\phi\left(x_{n}\right)\right)$, we have $p_{F}\left(\alpha_{n}^{-1} u\left(x_{n}\right)\right) \rightarrow 0$. Hence, there exist $z_{n} \in E$ such that $u\left(z_{n}\right)=u\left(\alpha_{n}^{-1} x_{n}\right)$ and $p_{E}\left(z_{n}\right) \rightarrow 0$. Then,

$$
1=p_{\hat{E}}\left(\alpha_{n}^{-1} \phi\left(x_{n}\right)\right)=p_{\hat{E}}\left(\phi\left(z_{n}\right)\right) \leqslant p_{E}\left(z_{n}\right) \rightarrow 0,
$$

a contradiction.

To prove that (2) implies (3), assume that (2) holds and $u(x) \in U_{F}(p)$. Then, $p_{\hat{E}}(\phi(x))<\alpha_{p}$, which means that there exists $z \in E$ such that $u(x)=u(z)$ and $p(z)<\alpha_{p}$. Hence, $u(x) \in u\left(U_{E}\left(p, \alpha_{p}\right)\right)$.

To prove that (3) implies (4), assume that (3) holds and $X$ is a $p$-open subset of $E$. Then, there exists $\alpha>0$ such that $U_{E}(p, \alpha) \subset X$, and, hence,

$$
u(E) \cap U_{F}\left(p, \alpha / \alpha_{p}\right) \subset u\left(U_{E}(p, \alpha)\right) \subset u(X),
$$

which shows that $u(X)$ is a $p$-open subset of $u(E)$. 
To prove that (4) implies (1), assume that (4) holds and $p_{F}\left(u\left(x_{i}\right)\right) \rightarrow 0$. Suppose that $p_{\hat{E}}\left(\phi\left(x_{i}\right)\right)>\alpha$ or some $\alpha>0$. It is obvious that $U_{E}(p, \alpha)$ is $p$-open and, hence, $u\left(U_{E}(p, \alpha)\right)$ is $p$-open. Therefore, $u\left(x_{i}\right) \in u\left(U_{E}(p, \alpha)\right)$, a contradiction.

We recall that a linear map $u: E \rightarrow F$ is said to be open if, for any neighborhood $U$ of zero in $E, u(U)$ is a neighborhood of zero in the subspace $u(E)$. It is easy to see that a continuous linear map $u: E \rightarrow F$ is open if and only if $\hat{u}: \hat{E} \rightarrow F$ is open.

(4.2). If $u: E \rightarrow F$ is open, there exists a directed calibration $\Gamma$ for $(E, F)$ such that $u \in L_{\Gamma}(E, F)$ and $u$ is $\Gamma$-open. If $u: E \rightarrow F$ is $\Gamma$-open for some calibration $\Gamma$ for $(E, F), u$ is open.

Proof. Let $u$ be open and $\Gamma^{\prime}$ be a calibration for $(E, F)$ such that $u \in$ $L_{\Gamma^{\prime}}(E, F)$. Then, for each $p^{\prime} \in \Gamma^{\prime}, u\left(U_{E}\left(p^{\prime}\right)\right)$ is an absolutely convex neighborhood of zero in the space $u(E)$. Hence, the Minkowski functional $\mu_{p^{\prime}}$ of $u\left(U_{E}\left(p^{\prime}\right)\right)$ is a continuous semi-norm on $u(E)$ and

$$
u\left(U_{E}\left(p^{\prime}\right)\right)=U_{u(E)}\left(\mu_{p^{\prime}}\right) .
$$

There exists a continuous extension of $\mu_{p^{\prime}}$ over $F$, which we shall denote by the same $\mu_{p}$. (Köthe (1967), Section 19, 4(2).) We define a new calibration $\Gamma$ for $(E, F)$ by

$$
\Gamma=\left\{p=\left(p_{E}^{\prime}, p_{F}^{\prime} \cup \mu_{p^{\prime}}\right): p^{\prime} \in \Gamma^{\prime}\right\} .
$$

Since $\Gamma^{\prime}$ could be chosen to be directed, this is also directed. Now, since

$$
u\left(U_{E}\left(p^{\prime}\right)\right)=U_{F}\left(\mu_{p^{\prime}}\right) \cap u(E),
$$

$p_{E}^{\prime}(x)<1$ implies $\mu_{p^{\prime}}(u(x))<1$, which means that there exists $\alpha_{p^{\prime}}>0$ such that

$$
\mu_{p}(u(x)) \leqslant \alpha_{p}, p_{E}^{\prime}(x) \text { for every } x \in E .
$$

Hence, for each $p \in \Gamma$, there exists $\alpha_{p}>0$ such that

$$
p_{F}(u(x))=\max \left\{p_{F}^{\prime}(u(x)), \mu_{p^{\prime}}(u(x))\right\}<\alpha_{p} p_{E}(x)
$$

for every $x \in E$, that is, $u \in L_{\Gamma}(E, F)$.

$u$ is $\Gamma$-open for this $\Gamma$, because

$$
u(E) \cap U_{F}(p) \subset u(E) \cap U_{F}\left(\mu_{p}\right) \subset u\left(U_{E}(p)\right)
$$

for every $p \in \Gamma$.

Conversely, if $u$ is $\Gamma$-open for a calibration $\Gamma$ for $(E, F)$, by considering $\hat{u}$ and the quotient calibration $\hat{\Gamma}$ if necessary, we may suppose that $u$ is injective. For a neighborhood $U$ of zero in $E$, there are $p_{i} \in \Gamma$ and $\alpha>0$ such that

$$
U_{E}\left(p_{1}, \alpha\right) \cap \cdots \cap U_{E}\left(p_{n}, \alpha\right) \subset U .
$$


Since $u$ is injective,

$$
u\left(\bigcap_{i=1}^{n} U_{E}\left(p_{i}, \alpha\right)\right)=\bigcap_{i=1}^{n} u\left(U_{E}\left(p_{i}, \alpha\right)\right),
$$

and, since $u$ is $\Gamma$-open, there exists $\beta>0$ such that

$$
u(E) \cap U_{F}\left(p_{i}\right) \subset u\left(U_{E}\left(p_{i}, \beta\right)\right) \text { if } 1<i<n .
$$

Hence,

$$
u(E) \cap \bigcap_{i=1}^{n} U_{F}\left(p_{i}, \alpha \beta^{-1}\right) \subset u(U)
$$

which shows that $u(U)$ is a neighborhood of zero in $u(E)$.

It is obvious that $p$-open maps are $p$ compatible. The precise relation between these two properties is given by the following statement.

(4.3). Let $\Gamma$ be a calibration for $(E, F), p \in \Gamma$ and $u \in L_{\mathrm{T}}(E, F)$. The following two conditions are equivalent:

(1) $u$ is p-open;

(2) $u$ is p-compatible and $u_{p}$ is open.

Proof. To prove that (1) implies (2), assume that $u$ is $p$-open. We only need to show that $u_{p}$ is open. By (4.1)(2), there exists $\alpha_{p}>0$ such that

$$
p_{E}(\phi(x)) \leqslant \alpha_{p} p_{F}(u(x)) \text { for every } x \in E .
$$

By extending this inequality over the $p$-completion, we have

$$
p_{E}\left(\phi_{p}(\mathbf{a})\right) \leqslant \alpha_{p} p_{F}\left(u_{p}(\mathbf{a})\right) \text { for every } \mathbf{a} \in E[p] .
$$

Hence, by (3.2)(6), we have

$$
p_{E}\left(\Phi_{p}(\mathbf{a})\right)<\alpha_{p} p_{F}\left(u_{p}(\mathbf{a})\right) \text { for every } \mathbf{a} \in E[p],
$$

which means that $u_{p}$ is open.

The converse can be proved by tracing the above proof in reverse order.

Let $\Gamma$ be a calibration for $(E, F)$ and $p \in \Gamma$. A continuous linear map $u$ : $E \rightarrow F$ is said to be almost $p$-open if there exists $\alpha_{p}>0$ such that

$$
\overline{u(E)} \cap B_{F}(p) \subset \overline{u\left(B_{E}\left(p, \alpha_{p}\right)\right)} \text {. }
$$

If $u$ is almost $p$-open for every $p \in \Gamma, u$ is said to be almost $\Gamma$-open. Obviously, $p$-open maps are almost $p$-open for every $p \in \Gamma$. 
We recall that a continuous linear map $u: E \rightarrow F$ is said to be almost open (or, nearly open in Köthe (1979), page 24) if, for any neighborhood $U$ of zero in $E$, $\overline{u(U)}$ is a neighborhood of zero in the subspace $\overline{u(E)}$ of $F$. Obviously, open maps are almost open.

By exactly the same method as in (4.2), we can prove the following fact.

(4.4). If $u: E \rightarrow F$ is almost open, there exists a directed calibration $\Gamma$ for $(E, F)$ such that $u$ is almost $\Gamma$-open. If $u: E \rightarrow F$ is almost $\Gamma$-open for some calibration $\Gamma$ for $(E, F), u$ is almost open.

A pair $(E, F)$ of locally convex spaces is said to be compatible if the following condition is satisfied: if a continuous linear map $u: E \rightarrow F$ is almost $\Gamma$-open for some calibration $\Gamma$ for $(E, F)$, then $u$ is $\Gamma$-compatible.

We recall that a locally convex space $E$ is called a Pták space if, for any locally convex space $F$, every almost open continuous linear map of $E$ into $F$ is open.

(4.5). If $E$ is a Pták space and $F$ is a locally convex space, the pair $(E, F)$ is compatible.

Proof. Assume that a continuous linear map $u: E \rightarrow F$ is almost $\Gamma$-open for some calibration $\Gamma$ for $(E, F)$. Then, for each $p \in \Gamma$, there exists $\alpha_{p}>0$ such that

$$
\overline{u(E)} \cap B_{F}(p) \subset \overline{u\left(B_{E}\left(p, \alpha_{p}\right)\right)} .
$$

By (4.4), $u$ is almost open and, hence, it is open. Therefore, there exists a continuous semi-norm $q_{p}$ on $F$ such that

$$
u(E) \cap U_{F}\left(q_{p}\right)=u\left(U_{E}\left(p, \alpha_{p}\right)\right)
$$

Now, assume that $u(x) \in U_{F}(p)$. Then, there exists $\alpha>0$ such that $p_{F}(u(x))<$ $\alpha<1$, and there exist $x_{\lambda} \in U_{E}\left(p, \alpha \alpha_{p}\right)$ such that $u\left(x_{\lambda}\right) \rightarrow u(x)$. Since $u\left(x_{\lambda}\right) \in$ $u\left(U_{E}\left(p, \alpha \alpha_{p}\right)\right), q_{p}\left(u\left(x_{\lambda}\right)\right)<\alpha$ and, hence, $q_{p}(u(x)) \leqslant 1$. Thus, $U_{F}(p) \subset U_{F}\left(q_{p}\right)$, which implies

$$
u(E) \cap U_{F}(p) \subset u\left(U_{E}\left(p, \alpha_{p}\right)\right) .
$$

Therefore, $u$ is $\Gamma$-open and, hence, $u$ is $\Gamma$-compatible.

(4.5) implies, in particular, the pair $(E[p], F[p])$ is a compatible pair. We use this fact in the proof of the following statement. 
(4.6). Assume that $\Gamma$ is a calibration for $(E, F)$ and $p \in \Gamma$. If $u \in L_{\Gamma}(E, F)$ is almost $p$-open, every $u_{p}: E[p] \rightarrow F[p]$ is open.

Proof. By the assumption, there exists $\alpha_{p}>0$ such that

$$
\overline{u(E)} \cap B_{F}(p) \subset \overline{u\left(B_{E}\left(p, \alpha_{p}\right)\right)} \text {. }
$$

Assume that

$$
\mathbf{b} \in \overline{u_{p}(E[p])} \cap U_{F}[p]
$$

then, since

$$
\overline{u_{p}(E[p])}=\overline{u_{p}\left(S_{p}(E)\right)},
$$

there exist $x_{i} \in E$ such that

$$
\lim _{i \rightarrow \infty} u_{p}\left(S_{p}\left(x_{i}\right)\right)=\mathbf{b} \text {. }
$$

We may suppose that $p_{F}\left(u\left(x_{i}\right)\right)<1$. Then, since $u\left(x_{i}\right) \in u(E) \cap B_{F}(p)$, there exist $z_{i} \in B_{E}\left(p, \alpha_{p}\right)$ such that $p_{F}\left(u\left(x_{i}\right)-u\left(z_{i}\right)\right) \rightarrow 0$, which implies

$$
u_{p}\left(S_{p}\left(z_{i}\right)\right) \rightarrow \mathbf{b} \text { and } u_{p}\left(S_{p}\left(x_{i}\right)\right) \in u_{p}\left(B_{E}\left[p, \alpha_{p}\right]\right) \text {. }
$$

Hence, $\mathrm{b} \in \overline{u_{p}\left(B_{E}\left[p, \alpha_{p}\right]\right)}$. This means that $u_{p}$ is almost open and, since $(E[p], F[p])$ is a compatible pair, $u_{p}$ is open.

We now have the exact relation between the almost $p$-openness and the $p$-openness, which follows from (4.3) and (4.6).

(4.7). Let $\Gamma$ be a calibration for $(E, F), p \in \Gamma$ and $u \in L_{\mathrm{r}}(E, F)$. Then $u$ is $p$-open if and only if $u$ is almost p-open and p-compatible.

Thus, we have the following statement which is the converse to (4.5).

(4.8). Let $E$ be a locally convex space and the pair $(E, F)$ be compatible for any locally convex space $F$. Then, $E$ is a Pták space.

Proof. Assume that a continuous linear map $u: E \rightarrow F$ is almost open. By (4.4), there exists a calibration $\Gamma$ for $(E, F)$ such that $u \in L_{T}(E, F)$ and $u$ is almost $\Gamma$-open. Since $(E, F)$ is a compatible pair, $u$ is $\Gamma$-compatible. By (4.7), $u$ is $\Gamma$-open and, by (4.2), $u$ is open. Hence, $E$ is a Pták space.

We are now in a position to introduce a wider class of locally convex spaces that is of essential importance to the theory of Treves calibration which will be developed in the next section. A locally convex space $E$ is said to be fully 
compatible if the following condition is satisfied: for any locally convex space $F$, if a continuous linear map $u: E \rightarrow F$ is almost open, then $u(E)$ is a closed subset of $F$.

(4.9). Every Pták space is fully compatible.

Proof. Let $E$ be a Pták space and $F$ be a locally convex space. When $u$ : $E \rightarrow F$ is an almost open continuous linear map, it is open and, since $E / \operatorname{ker}(u)$ is complete, $u(E)$ is closed (Köthe (1979), page 7).

As in Example 2 in Section 2, we denote the completion of $E$ by $\tilde{E}$ and let $\psi$ : $E \rightarrow \tilde{E}$ be the canonical embedding.

(4.10). Fully compatible spaces are complete.

Proof. Let $E$ be fully compatible. Since $\psi: E \rightarrow \tilde{E}$ is an open map, $\psi(E)$ is closed. Since it is dense, $\psi(E)=\tilde{E}$ and, hence, $E$ is complete.

Let $E$ and $F$ be locally convex spaces and $M$ be a closed linear subspace of $E$. Let $\Gamma$ be a calibration for $(E, F)$ and $\Gamma^{\prime}$ be a directed calibration for $(E / M, F)$. Then, for each $p \in \Gamma$, since $\hat{p}_{E}$, the semi-norm on $E / M$ defined by $p_{E}$ as in Example 1 in Section 2, is continuous, there exists $p^{\prime} \in \Gamma^{\prime}$ and $\alpha>0$ such that $\hat{p}_{E}<\alpha\left(p^{\prime}\right)_{\hat{E}}$. Then, for $\hat{p}_{F}=p_{F} \cup\left(p^{\prime}\right)_{F}$, we have a calibration

$$
\hat{\Gamma}=\left\{\hat{p}=\left(\hat{p}_{E}, \hat{p}_{F}\right): p \in \Gamma\right\}
$$

for $(E / M, F)$ such that

$$
B_{\hat{E}}(\hat{p}) \supset B_{\hat{E}}\left(p^{\prime}, \alpha^{-1}\right) \quad \text { and } \quad B_{F}(\hat{p}) \subset B_{F}\left(p^{\prime}\right) .
$$

Therefore, if a continuous linear map $v: E / M \rightarrow F$ is almost $\Gamma^{\prime}$-open, then it is almost $\hat{\Gamma}$-open, and $\hat{\Gamma}$ is a calibration for $(E / M, F)$ such that $(\hat{\Gamma})_{E / M}$ is defined from $\Gamma_{E}$ by taking the quotients.

(4.11). If $E$ is a fully compatible space and $M$ is a closed linear subspace of $E$, the quotient space $E / M$ is fully compatible.

Proof. Let $v: E / M \rightarrow F$ be a continuous linear map which is almost open. There exists a directed calibration $\hat{\Gamma}$ for $(E / M, F)$ such that $v$ is almost $\hat{\Gamma}$-open. We may suppose that there exists a calibration $\Gamma$ for $(E, F)$ such that $(\hat{p})_{F}=p_{F}$ and

$$
(\hat{p})_{E / M}(\phi(x))=\inf \left\{p_{E}(z): x-z \in M\right\}
$$


where $\phi: E \rightarrow E / M$ is the canonical map. Then, the continuous linear map $u$ : $E \rightarrow F$ defined by $u=v \circ \phi$ is almost $\Gamma$-open, because

$$
v\left(B_{\hat{E}}(\hat{p})\right)=u\left(B_{E}(p)\right) \text {. }
$$

Therefore, $v(\hat{E})=u(E)$ is closed.

Finally, either (2.6) or (2.7) implies the following fact.

(4.12). Let $E$ be a fully compatible space and $F$ be a locally convex space. Let $\Gamma$ be a calibration for $(E, F)$ such that $\Gamma_{F}$ is gauged and $u: E \rightarrow F$ be a continuous linear map which is almost $\Gamma$-open. Then, if every $u_{p}: E[p] \rightarrow F[p]$ is surjective, $u$ is surjective.

\section{Treves calibrations}

We use the Treves' notation $\operatorname{Spec}(E)$ to denote the set of all continuous semi-norms of a locally convex space $E$. When $F$ is another locally convex space, a continuous linear map $u: E \rightarrow F$ defines a map:

$$
u_{*}: \operatorname{Spec}(F) \rightarrow \operatorname{Spec}(E): q \mapsto q \circ u \text {. }
$$

Treves (1967) called a pair $(E, F) u$-convex if, for each $p \in \operatorname{Spec}(E)$, there exists $q \in \operatorname{Spec}(F)$ such that $\operatorname{ker}(q) \subset \operatorname{ker}(r)$ whenever $u_{*} r<p$. He also called a continuous linear map $u: E \rightarrow F$ semiglobally surjective if, for each $q \in \operatorname{Spec}(F)$ and $y \in F$, there exists $x \in E$ such that $q(y-u(x))=0$. The so-called Treves surjectivity theorem states that, if $E$ and $F$ are Fréchet spaces, $(E, F)$ is $u$-convex and $u$ is semiglobally surjective, then $u$ is surjective. This generalizes an existence theorem for some partial differential equations. In the proof of this theorem, Treves noted that, in general, if $(E, F)$ is $u$-convex and $u$ is semiglobally surjective,

$$
\sup \left\{r(y): u_{*} r \leqslant p, r \in \operatorname{Spec}(F)\right\}<+\infty
$$

for every $p \in \operatorname{Spec}(E)$ and $y \in E$, and, when $F$ is barrelled, the following condition is satisfied: for each $p \in \operatorname{Spec}(E)$ there exists $q \in \operatorname{Spec}(F)$ such that $r<q$ whenever $u_{*} r \leqslant p$ and $r \in \operatorname{Spec}(F)$. We use this fact to define the Treves calibration for $(E, F)$.

A calibration $\Gamma$ for $(E, F)$ is called a Treves calibration for $(E, F)$ defined by $u$ if the following condition is satisfied: if $q \in \operatorname{Spec}(F)$ and $u_{*} q \leqslant p_{E}$, then $q<p_{F}$. 
By the Hahn-Banach theorem, the above condition is equivalent to the following: if $y^{\prime} \in F^{\prime}$ (the dual of $F$ ) and $u_{*}\left|y^{\prime}\right| \leqslant p_{E}$, then $\left|y^{\prime}\right|<p_{F}$, where

$$
\left|y^{\prime}\right|(y)=\left|y^{\prime}(y)\right| \text { for every } y \in F \text {. }
$$

It is obvious that the quotient calibration in Example 1 in Section 2 is a Treves calibration for $(E, \hat{E})$ defined by $\phi$ and the completion calibration in Example 2 there is a Treves calibration for $(E, \tilde{E})$ defined by $\psi$.

(5.1). A calibration $\Gamma$ for $(E, F)$ is a Treves calibration defined by $u$ if and only if $B_{F}(p) \subset \overline{u\left(B_{E}(p)\right)}$ for every $p \in \Gamma$.

Proof. Assume that $\Gamma$ is a Treves calibration for $(E, F)$ defined by $u$ and $y \in B_{F}(p)$. If $y$ does not belong to the absolutely convex closed subset $\overline{u\left(B_{E}(p)\right)}$, there exists $y^{\prime} \in F^{\prime}$ such that $\left|y^{\prime}\right|(y)>1$ and $\left|y^{\prime}\right|<1$ on $u\left(B_{E}(p)\right)$, but the latter implies $u_{*}\left|y^{\prime}\right| \leqslant p_{E}$, which should lead to $\left|y^{\prime}\right|<p_{F}$, a contradiction.

Conversely, assume that $B_{F}(p) \subset \overline{u\left(B_{E}(p)\right)}$ for all $p \in \Gamma$ and $u_{*} r<p_{E}$ for some $r \in \operatorname{Spec}(F)$. Then, $u\left(B_{E}(p)\right) \subset B_{F}(r)$, which implies $B_{F}(p) \subset B_{F}(r)$ and, hence, $r \leqslant p_{F}$.

It is now clear from (4.4) that a continuous linear map $u: E \rightarrow F$ defines a Treves calibration for $(E, F)$ if and only if $u$ is almost open and $\overline{u(E)}=F$.

(5.2). Let $E$ be a locally convex space. Then, every continuous linear map $u$ of $E$ into arbitrary locally convex space $F$ which defines a Treves calibration for $(E, F)$ is surjective if and only if $E$ is fully compatible.

Proof. Assume that $u: E \rightarrow F$ is a continuous linear map which is almost open. Then, by (4.4), there exists a calibration $\Gamma$ for $(E, F)$ such that, for each $p \in \Gamma$, there exists $\alpha_{p}>0$ such that

$$
\overline{u(E)} \cap B_{F}(p) \subseteq \overline{u\left(B_{E}\left(p, \alpha_{p}\right)\right)} \text {. }
$$

Now, set

$$
\bar{p}_{E}=p_{E} \quad \text { and } \quad \bar{p}_{F}=\alpha_{p}^{-1} p_{F} / \overline{u(E)} \text { (the restriction to } \overline{u(E)} \text { ). }
$$

Then, $\bar{\Gamma}=\left\{\left(\bar{p}_{E}, \bar{p}_{F}\right): p \in \Gamma\right\}$ is a Treves calibration for $(E, \overline{U(E)})$. Therefore, by the assumption, $u: E \rightarrow \overline{u(E)}$ must be surjective, which means that $u(E)$ is closed. The converse is obvious.

We shall add a few facts about the case when $E$ is not necessarily fully compatible. 
(5.3). If a continuous linear map $u: E \rightarrow F$ defines a Treves calibration $\Gamma$ for $(E, F)$ and $u \in L_{T}(E, F)$, then every $u_{p}: E[p] \rightarrow F[p]$ is surjective. In fact,

$$
U_{F}[p] \subset u_{p}\left(U_{E}[p]\right) \text { for every } p \in \Gamma \text {. }
$$

Proof. It follows from the assumption that the calibration $\Gamma_{p}$ which consists of a single norm map $p=\left(p_{E}, p_{F}\right)$ is a Treves calibration for $(E[p], F[p])$ defined by $u_{p}$. It is obvious that $u_{p}$ is $\Gamma_{p}$-compatible. Hence, by (4.7), $u_{p}$ is $\Gamma_{p}$-open, which, in conjunction with (5.2), implies the required result.

Hence, the following fact is implies by (2.1).

(5.4). Let $\Gamma$ be a Treves calibration for $(E, F)$ defined by $u$ and $u \in L_{\mathrm{T}}(E, F)$. Then, $u$ is surjective if and only $u$ is $S$-resonant.

The map $u=\psi \circ \phi$ considered in Example 3 in Section 2 defines a Treves calibration and is not surjective. This fact shows that a complete space which has a non-complete quotient space by a closed linear subspace is not fully compatible.

\section{Theorems of M. Eidelheit and E. Borel}

Let $\Gamma_{E}$ be a calibration for a locally convex space $E$. We consider an index set $\Lambda$, a family $\left\{F_{\lambda}: \lambda \in \Lambda\right\}$ of Banach spaces and a family $\left\{u_{\lambda}: \lambda \in \Lambda\right\}$ of continuous linear maps of $E$ into $F_{\lambda}$. Then, we have a map

$$
u: E \rightarrow F: x \mapsto\left(u_{\lambda}(x)\right)
$$

of $E$ into the product space $F=I I F_{\lambda}$.

A theorem of Eidelheit (Köthe (1979), page 126) states that, when $E$ is $a$ Fréchet space with a calibration $\left(p_{k}\right)$ and $u_{i} \in E^{\prime}$ (the dual of $E$ ), the system of equations:

$$
u_{i}(x)=\eta_{i}, \quad i=1,2, \ldots,
$$

has a solution $x$ for every sequence $\left(\eta_{i}\right)$ of real numbers if and only if the following two conditions are satisfied:

(E.1) $u_{i}$ are linearly independent;

(E.2) for each $p_{k}$ there exists a constant $n_{k}$ such that, if

$$
\sum_{i=1}^{n} \eta_{i} u_{i} \in\left(E, p_{k}\right)^{\prime} \text { and } \eta_{n} \neq 0,
$$

then $n<n_{k}$, where $\left(E, p_{k}\right)^{\prime}$ is the dual of the semi-normed space $\left(E, p_{k}\right)$. 
In other words, this theorem states that, when $E$ is a Fréchet space, $\Lambda$ is a countable set and $F_{\lambda}=R$, the map $u$ is surjective if and only if the conditions (E.1) and (E.2) are satisfied. We shall prove that these conditions are in fact equivalent to the existence of a Treves calibration for $(E, F)$ defined by $u$. As a preparation, we shall express these conditions in a more convenient form, which, in the following statement (6.1), will be written in the form applicable to the general case described in the beginning of this section, where $\eta_{\lambda}$ are continuous linear functionals on the Banach spaces $F_{\lambda}$, whose norms will be denoted by $\|\cdot\|_{\lambda}$.

(6.1). When $E$ is a Fréchet space with a calibration $\Gamma_{E}=\left\{p_{k}: k=1,2, \ldots\right\}$ and $u_{i} \in E^{\prime}(i=1,2, \ldots)$, the conditions (E.1) and (E.2) together is equivalent to the following condition:

(T) for each $p \in \Gamma_{E}$ there exists a finite set $\Lambda_{p}$ of positive integers and a positive constant $\alpha_{p}$ such that, if

$$
\left|\sum_{i=1}^{n} \eta_{\lambda_{i}} \circ u_{\lambda_{i}}\right| \leqslant p \text { and } \eta_{\lambda_{i}} \neq 0
$$

then $\lambda_{i} \in \Lambda_{p}$ and $\left\|\eta_{\lambda_{i}}\right\|_{\lambda_{i}} \leqslant \alpha_{p}$ for all $i$ between 1 and $n$.

Proof. Assume that the conditions (E.1) and (E.2) hold, $p_{k} \in \Gamma_{E}$ and let $\Lambda_{k}=\Lambda_{p_{k}}$ be the set of integers between 1 and $n_{k}$. Then, since $\left\{u_{i}: 1<i<n_{k}\right\}$ is linearly independent, we can find $e_{k, i} \in E$ such that

$$
u_{i}\left(e_{k, i}\right)=1 \text { and } u_{i}\left(e_{k, j}\right)=0 \text { if } i \neq j, 1 \leqslant i, j \leqslant n_{k} .
$$

We set

$$
\alpha_{k}=\max \left\{p_{k}\left(e_{k, i}\right): 1 \leqslant i \leqslant n_{k}\right\}
$$

Now, assume that

$$
\left|\sum_{i=1}^{n} \eta_{i} u_{i}(x)\right| \leqslant p_{k}(x) \quad \text { for every } x \in E
$$

and $\eta_{n} \neq 0$. Then, by (E.2), $n \in \Lambda_{k}$ and

$$
\left|\eta_{i}\right|=\left|\sum_{i=1}^{n} \eta_{i} u_{i}\left(e_{k, i}\right)\right| \leqslant p_{k}\left(e_{k, i}\right) \leqslant \alpha_{k}
$$

for all $i$ between 1 and $n$. Hence, the condition (T) is satisfied.

Conversely, assume that the condition (T) is satisfied. To show that (E.1) is satisfied, assume that

$$
\sum_{i=1}^{n} \eta_{i} u_{i}=0
$$


Then, for each $k$, since

$$
\left|\sum_{i=1}^{n} \xi \eta_{i} u_{i}(x)\right| \leqslant p_{k}(x) \text { for all } \xi>0 \text { and } x \in E \text {, }
$$

we have $\left|\xi \eta_{i}\right|<\alpha_{k}$ for all $\xi>0$, or, $\eta_{i}=0$.

To show that (E.2) is satisfied, let

$$
n_{k}=\max \left\{n: n \in \Lambda_{k}\right\} \text {. }
$$

Then, if

$$
\sum_{i=1}^{n} \eta_{i} u_{i} \in\left(E, p_{k}\right)^{\prime} \quad \text { and } \quad \eta_{n} \neq 0
$$

there exists $\alpha>0$ such that

$$
\left|\sum_{i=1}^{n} \alpha \eta_{i} u_{i}\right| \leqslant p_{k} \text { and } \alpha \eta_{n} \neq 0
$$

which implies $n \leqslant n_{k}$.

Now, we are ready to consider the general case.

(6.2). Let $E$ be a locally convex space, $F_{\lambda}$ be Banach spaces, $u_{\lambda}: E \rightarrow F_{\lambda}$ be continuous linear maps and

$$
u: E \rightarrow F=\Pi F_{\lambda}: x \mapsto\left(u_{\lambda}(x)\right) .
$$

Then, there exists a Treves calibration for $(E, F)$ defined by $u$ if and only if the condition $(\mathrm{T})$ holds for some calibration for $E$.

Proof. Assume that $\Gamma$ is a Treves calibration for $(E, F)$ defined by $u$. Then, for each $p \in \Gamma$, since $p_{F}$ is a continuous semi-norm on the product space $F=\Pi F_{\lambda}$, there exist a finite set $\Lambda_{p}$ and a positive constant $\alpha_{p}$ such that

$$
p_{F} \leqslant \sum\left\{\alpha_{p}\|\cdot\|_{\lambda}: \lambda \in \Lambda_{p}\right\}
$$

Now, assume that, for some $\eta_{\lambda_{i}} \in F_{\lambda_{i}}^{\prime}$, we have

$$
\left|\sum_{i=1}^{n} \eta_{\lambda_{i}} \circ u_{\lambda_{i}}\right| \leqslant p_{E} \quad \text { and } \quad \eta_{\lambda_{i}} \neq 0 \text {. }
$$

Let $y^{\prime}$ be the continuous linear functional on $F$ defined by

$$
y^{\prime}(y)=\sum_{i=1}^{n} \eta_{\lambda_{i}}\left(\xi_{\lambda}\right) \text { for } y=\left(\xi_{\lambda}\right) \in F .
$$

Then, the above inequality is equivalent to $u_{*}\left|y^{\prime}\right| \leqslant p_{E}$. Hence, we should have $\left|y^{\prime}\right|<p_{F}$. Therefore, $\lambda_{n} \in \Lambda_{p}$ and $\left\|\eta_{\lambda_{l}}\right\| \leqslant \alpha_{p}$ for all $i$ between 1 and $n$. 
Conversely, assume that the condition (T) is satisfied for a calibration $\Gamma_{E}$ for $E$. Then, for each $p_{E} \in \Gamma_{E}$, we set

$$
p_{F}(y)=\sum\left\{\alpha_{p}\left\|\xi_{\lambda}\right\|_{\lambda}: \lambda \in \Lambda_{p}\right\} \quad \text { for } y=\left(\xi_{\lambda}\right) \in F,
$$

and let

$$
\Gamma=\left\{p=\left(p_{E}, p_{F}\right): p_{E} \in \Gamma_{E}\right\} .
$$

Since, for each $\lambda \in \Lambda$, there exists $p_{E} \in \Gamma_{E}$ such that $\lambda \in \Lambda_{p}$ and

$$
p_{F}\left(\xi_{(\lambda)}\right)=\alpha_{p}\left\|\xi_{\lambda}\right\|_{\lambda} \text { for all } \xi_{\lambda} \in F_{\lambda},
$$

where $\xi_{(\lambda)}$ is the element of $F$ whose single non-zero coordinate is $\xi_{\lambda}, \Gamma$ is a calibration for $(E, F)$. To prove that this is a Treves calibration defined by $u$, assume that $y^{\prime} \in F^{\prime}$. Since $F^{\prime}$ is the locally convex direct sum of $F_{\lambda}^{\prime}$, it has only finite number of non-zero coordinates; let $\Lambda\left(y^{\prime}\right)$ be the set of such coordinates. Then, $y^{\prime}$ is in the form of

$$
y^{\prime}(y)=\sum\left\{\eta_{\lambda}\left(\xi_{\lambda}\right): \lambda \in \Lambda\left(y^{\prime}\right)\right\} \quad \text { for all } y=\left(\xi_{\lambda}\right) \in F .
$$

Therefore, $u_{*}\left|y^{\prime}\right| \leqslant p_{E}$ is equivalent to

$$
\left|\Sigma\left\{\eta_{\lambda} \circ u_{\lambda}: \lambda \in \Lambda\left(y^{\prime}\right)\right\}\right| \leqslant p_{E} \text { and } \eta_{\lambda} \neq 0 \text { for } \lambda \in \Lambda\left(y^{\prime}\right) \text {. }
$$

Hence, by the condition (T), we have $\Lambda\left(y^{\prime}\right) \subset \Lambda_{p}$ and $\left\|\eta_{\lambda}\right\|_{\lambda} \leqslant \zeta_{p}$ if $\lambda \in \Lambda\left(y^{\prime}\right)$. Then, for $y=\left(\xi_{\lambda}\right) \in F$,

$$
\left|y^{\prime}\right|(y)=\left|\Sigma\left\{\eta_{\lambda}\left(\xi_{\lambda}\right): \lambda \in \Lambda\left(y^{\prime}\right)\right\}\right| \leqslant \Sigma\left\{\alpha_{p}\left\|\xi_{\lambda}\right\|_{\lambda}: \lambda \in \Lambda\left(y^{\prime}\right)\right\} \leqslant p_{F}(y) .
$$

Thus, the following proposition is an immediate consequence of (5.2).

(6.3). Let $E$ be fully compatible, $F_{\lambda}$ be Banach spaces and $u_{\lambda}: E \rightarrow F_{\lambda}$ be continuous lienar maps. Then, if the condition $(\mathrm{T})$ is satisfied, the system of equations $u_{\lambda}(x)=\xi_{\lambda}$ has a solution for every $\left(\xi_{\lambda}\right) \in F$.

We use this theorem to prove a generalized form of a theroem of E. Borel. Let $X$ and $Y$ be Banach spaces. When $f: X \rightarrow Y$ is a $C^{\infty}$-map on $X$, the $n$-th derivative $f^{(n)}(x)$ of $f$ at $x \in X$ belongs to the Banach space $L_{s}^{n}(X, Y)$ of all symmetric $n$-linear continuous maps of $X$ into $Y$ equipped with the norm

$$
\|v\|=\sup \{\|v(x, \ldots, x)\|:\|x\| \leqslant 1\} .
$$

Let $C_{B}^{\infty}(X, Y)$ be the space of all $C^{\infty}$-maps $f: X \rightarrow Y$ which satisfy the following condition:

$$
p_{k, n}(f)=\sup \left\{\left\|f^{(i)}(x)\right\|: 0 \leqslant i \leqslant k,\|x\| \leqslant n\right\}<+\infty
$$

for all $k>0$ and $n \geqslant 1$. Then, $C_{B}^{\infty}(X, Y)$ is a Fréchet space with $\left(p_{k, n}\right)$ as its calibration. 
We consider a sequence $\left(u_{i}\right)$ of maps defined by

$$
u_{i}: C_{B}^{\infty}(X, Y) \rightarrow L_{s}^{i}(X, Y): f \mapsto f^{(i)}(0)
$$

for every $i \geqslant 0$. Then, since

$$
\left\|u_{i}(f)\right\| \leqslant p_{i, n}(f)
$$

every $u_{i}$ is a continuous linear map. Then, for $E=C_{B}^{\infty}(X, Y), F_{i}=L_{s}^{i}(X, Y)$ and $F=\prod F_{i}$, we define a map:

$$
u: E \rightarrow F: f \mapsto\left(f(0), f^{\prime}(0), \ldots, f^{(i)}(0), \ldots\right) .
$$

The theorem of E. Borel states that $u$ is surjective when $X$ is a finite-dimensional Euclidean space and $Y$ is that of complex numbers. Treves (1967), page 58, gave a proof of this theorem as an application of his surjectivity theorem. Dieudonné (1969), page 192, has a version of the thoerem when $X=R$ and $Y$ is a Banach space. Colombeau (1979) has proved that the map $u$ is surjective when $X$ is a locally convex space with some additional assumptions and $Y$ is a Fréchet space. The essential part of his proof is the fact that the theorem holds when $X$ is a pre-Hilbert space and $Y$ is a Banach space.

A Banach space $X$ is said to be $C_{B}^{\infty}$-normal (Yamamuro (1974), page 106) if, for arbitrary disjoint closed subsets $A$ and $B$, there exists $h \in C_{B}^{\infty}(X, R)$ such that $0<h<1, h=0$ on $A$ and $h=1$ on $B$. The following lemma is essentially due to Colombeau (1979), page 98.

(6.4). Let $X$ be a $C_{B}^{\infty}$-normal Banach space and $Y$ be a Banach space. Let $p_{k, n}$ be a semi-norm on $C_{B}^{\infty}(X, Y)$ as defined above. Assume that $f \in C_{B}^{\infty}(X, Y)$ and $f^{(i)}(0)=0$ if $0 \leqslant i \leqslant k$. Then, for any $\varepsilon>0$, there exists $g \in C_{B}^{\infty}(X, Y)$ such that $g=0$ in a neighborhood of zero and $p_{k, n}(f-g)<\varepsilon$.

Combining (6.3) and (6.4), we have the following fact which again due essentially to Colombeau (1979), page 100.

(6.5). When $X$ is a $C_{B}^{\infty}$-normal Banach space and $Y$ is a Banach space, the map $u$ is surjective.

Proof. Since $E=C_{B}^{\infty}(X, Y)$ is a Fréchet space and $F_{i}=L_{x}^{i}(X, Y)$ are Banach spaces, we only need to show that the condition (T) is satisfied. Assume that, for some $p_{k, n}$, there exist non-zero $\eta_{i} \in F_{i}^{\prime}$ such that

$$
\left|\sum_{i=0}^{m} \eta_{j} \circ u_{i}\right| \leqslant p_{k, n}
$$


Let $v_{m}$ be an arbitrary element of $F_{m}$ such that $\eta_{m}\left(v_{m}\right) \neq 0$ and consider the polynomial $P_{m}$ defined by $v_{m}$ :

$$
P_{m}(x)=v_{m}(x, \ldots, x) .
$$

Then,

$$
P_{m}^{(i)}(0)=0 \quad \text { if } i \neq m \text { and } P_{m}^{(m)}(0)=m ! v_{m} .
$$

Therefore, if $m>k$, the map $g_{m}$ determined by (6.4) with $\varepsilon<m !\left|\eta_{m}\left(v_{m}\right)\right|$, we set $f_{m}=P_{m}-g_{m}$ and we obtain

$$
\left|\sum_{i=0}^{m} \eta_{i} u_{i}\left(f_{m}\right)\right| \leqslant p_{k, n}\left(f_{m}\right)
$$

or, $m !\left|\eta_{m}\left(v_{m}\right)\right| \leqslant \varepsilon$, a contradiction. Hence, $m \leqslant k$. Furthermore, it is easy to see that

$$
\left\|\eta_{i}\right\| \leqslant \alpha_{k, n} \text { if } 0 \leqslant i \leqslant m,
$$

where

$$
\alpha_{k, n}=\max \left\{n^{k}, k n^{k-1}, \ldots, k !\right\}
$$

\section{References}

J. F. Colombeau (1979), 'Infinite dimensional $C^{\infty}$-mappings with a given sequence of derivatives at a given point', J. Math. Anal. Appl. 71, 95-104.

J. Dieudonné (1969), Foundations of modern analysis, (Academic Press, New York and London).

G. Köthe (1967), Topological vector spaces I, (Springer-Verlag, Berlin, Heiderberg, New York).

G. Köthe (1979), Topological vector spaces II, (Springer-Verlag, Berlin, Heiderberg, New York).

B. Malgrange (1966), Ideals of differentiable functions, (Studies in Mathematics 3, Oxford University Press, London).

R. Mennicken and B. Sagraloff (1980), 'Characterization of nearly openness', J. Reine Angew. Math. 313, $105-115$.

F. Treves (1967), Locally convex spaces and linear partial differential equations, (Springer-Verlag, Berlin, Heiderberg, New York).

S. Yamamuro (1974), Differential calculus in topological linear spaces, (Lecture Notes in Mathematics 374, Springer-Verlag, Berlin, Heiderberg, New York).

S. Yamamuro (1975), 'A differentiation in locally convex spaces', Bull. Austral. Math. Soc. 12, 183-209.

S. Yamamuro (1978), 'A note on Omori-Lie groups', Bull. Austral. Math. Sac. 19, 333-349.

S. Yamamuro (1979), A theory of differentiation in locally convex spaces, (Mem. Amer. Math. Soc. 1, no. 212, Providence, R.I.). 
S. Yamamuro (1979a), 'A note on the Omega lemma', Bull. Austral. Math. Soc. 20, 421-435.

S. Yamamuro (1980), 'Notes on the inverse mapping theorem in locally convex spaces', Bull. Austral. Math. Sac. 21, 419-461.

Department of Mathematics

Institute of Advanced Studies

Australian National University

Canberra A.C.T.

Australia 